\title{
MyD88 and Cancer
}

\author{
Indrani Barman ${ }^{1}$ and Manash Pratim Sarma ${ }^{2 *}$ \\ ${ }^{1}$ Department of Biochemistry, Assam down town University, Panikhaiti, Guwahati, Assam, India; ${ }^{2}$ Department of Biotechnology, Assam \\ down town University, Panikhaiti, Guwahati, Assam, India
}

\begin{abstract}
Cancer is a condition characterized by uncontrolled growth of cells that leads to formation of lumps or masses of tissues called tumors. There are different types of cancer, each of which are basically linked to the type of cell that it affects. Cancer may be caused by hereditary factors, environmental factors, carcinogenic agents and exposure to radiation, among other sources. Often referred to as the "Silent Killer," cancer is one of the deadliest diseases affecting human populations worldwide. In India, approximately 1 million cases of cancer are detected annually and added to the total pool of patients suffering from this disease. Cancer cases are rising at an alarming rate over the last decade in India, with increases being particularly high in the North Eastern part of the country. Head and neck cancer leads the list in northeast India, as per reports from the National Cancer Registries and ICMR. Researchers all over the globe, however, have adopted numerous ways to study the molecular mechanisms associated with cancer biology and all their approaches can be broadly summarized under genomic and proteomic studies. Many pathways have been proposed to reflect the mechanisms involved in cancer. However, the one mediated by P53 and the myeloid differentiation factor 88 (MyD88) has gained significant attention. P53 is a tumor suppressor protein, encoded by the gene TP53 in humans. It acts by regulating the cell cycle via integration of various signals linked to cell life and death, and as such plays a crucial role in preventing cancer. It also activates the programmed cell death pathway, or apoptosis, thereby arresting cell growth. While a substantial amount of data has been published on $\mathrm{p} 53$, the MyD88 pathway remains much less studied and largely unknown; research efforts are ongoing with the aim of elucidat-
\end{abstract}

Keywords: MyD88; IL-1; IRAK; TRIF; TRAF6; TBK1; TIRAP; TIR; TNF- $\alpha$; IL-1b; IL-6; IP-10; IFN- $\gamma$; NF- $\kappa B$.

Abbreviations: MyD88, myeloid differentiation factor 88; IL, interleukin; IRAK, IL1 receptor-associated kinase; TIR, Toll-like receptor; TRIF, TIR-domain-containing adapter inducing interferon- $\beta$; TRAF6, TNF Receptor-Associated Factor 6; TBK1,

TANK binding kinase 1; TIRAP, TIR domain-containing adaptor protein; TNF- $\alpha$, tumor necrosis factor $\alpha$; IL-1b, Interleukin-1 beta; IL-6, Interleukin 6; IP-10, interferon-inducible protein 10; IFN, interferon; IFN- $\gamma$, interferon gamma; NF- $\mathrm{kB}$, nuclear factor-kappaB; TRAM, TRIF-related adaptor molecule; IRF3, interferon regulatory factor 3; IKK, IKB kinase; Mal, MyD88-adaptor-like; TNFR, tumor necrosis factor receptor; MAPK, mitogen-activated protein kinase; TAB, TGF-beta activated kinase 1/MAP3K 7 binding protein; TAK, transforming growth factor beta-activated kinase; NEMO, NF-kappa-B essential modulator; JNK, c-Jun N-terminal kinase; TICAM-1, TIR domain-containing adaptor molecule-1; ABC-DLBCL, activated B cell type-diffuse large B cell lymphoma; WM, Waldenström's macroglobulinemia; CLL, chronic lymphocytic leukemia; CBCL, cutaneous diffuse large B cell lymphoma; PCNSL, primary central nervous system lymphoma; DD, death domain

Received: 15 March 2016; Revised: 8 April 2016; Accepted: 11 May 2016

DOI: 10.14218/ERHM.2016.00003

*Correspondence to: Manash Pratim Sarma, Department of Biotechnology, Assam Down Town University, Panikhaiti, Guwahati, Assam, India. Tel: +91-8255075275, E-mail: manash3268@gmail.com ing the signaling mechanism of MyD88 in cancer. In this article, emphasis is laid on discussing the signaling pathways associated with MyD88.

\section{Introduction}

MyD88 was first characterized for its role in the process of hematopoiesis, and defined as an encoded protein of the myeloid differentiation primary response gene. ${ }^{1}$ Since then, MyD88 has been determined to play a significant role in exerting innate immunological responses in mammals. ${ }^{2}$ The pioneer study on MyD88 was carried out by taking into account the amino acid homology that exists between MyD88 and the cytoplasmic domains of Drosophila Toll and the mammalian interleukin (IL)-1 receptors, which further illustrated that "MyD88 may be defined as a family of signal transduction molecules with an ancestral function in the activation of the immune system."

MyD88 is now known to be a critical adaptor molecule that bridges IL-1R1 with the IL-1 receptor-associated kinase (IRAK) complex, thereby highlighting its role as an immunological pillar. ${ }^{3}$ This adaptor molecule exhibits a vital character in directing innate immune signaling through the Toll-like receptor (TLR) members and the IL-1 family, and can function in both pro- and anti-tumorigenic responses, as shown in various cancer model systems. ${ }^{4}$ The MyD88 protein itself acts as an anchor to direct protein signaling of TLR/IL-1R, ${ }^{5}$ as well as of receptors with IFN- $\gamma$ in the innate immunological response. The modular composition of MyD88 includes a death domain (DD) at its $\mathrm{NH}_{2}$ terminus and a Toll/IL-1R (TIR) domain at the $\mathrm{COOH}$ terminus. ${ }^{6}$

MyD88 and the TIR-domain-containing adapter inducing interferon- $\beta$ (TRIF) act as crucial adaptors that bind TLRs and their intracellular domains, thereby activating the pro-inflammatory response. In a recent study conducted in rats suffering from ovarian cancer, the TLR4-induced MyD88 and TRIF-dependent pathways were shown to be attenuated by melatonin. ${ }^{7}$ The various cytokines that are expressed, such as tumor necrosis factor- $\alpha$ (TNF- $\alpha$ ), IL$1 \beta$, IL-6, interferon-inducible protein 10 (IP-10) and IFN- $\gamma$, are mediated and characterized by a dual coordination of MyD88 and TRIF through various activated transcriptional factors, including NF- $\kappa$ B, AP- 1 and IRF- $3 .{ }^{8}$ In TLR-dependent signaling pathways, MyD88 was first recognized as the common adapter that leads to the activation of innate immunity. ${ }^{9}$

Various experimental models have been used to evidence the involvement of MyD88 in cancer. Its roles include promotion of tumor formation and cancer-assisted inflammation. ${ }^{10}$ Inflammatory cytokines are induced with the help of MyD88, and the adaptor that contains a TIR domain, via action of all the TLRs. The first of its kind to be described, MyD88, an intracellular adaptor 


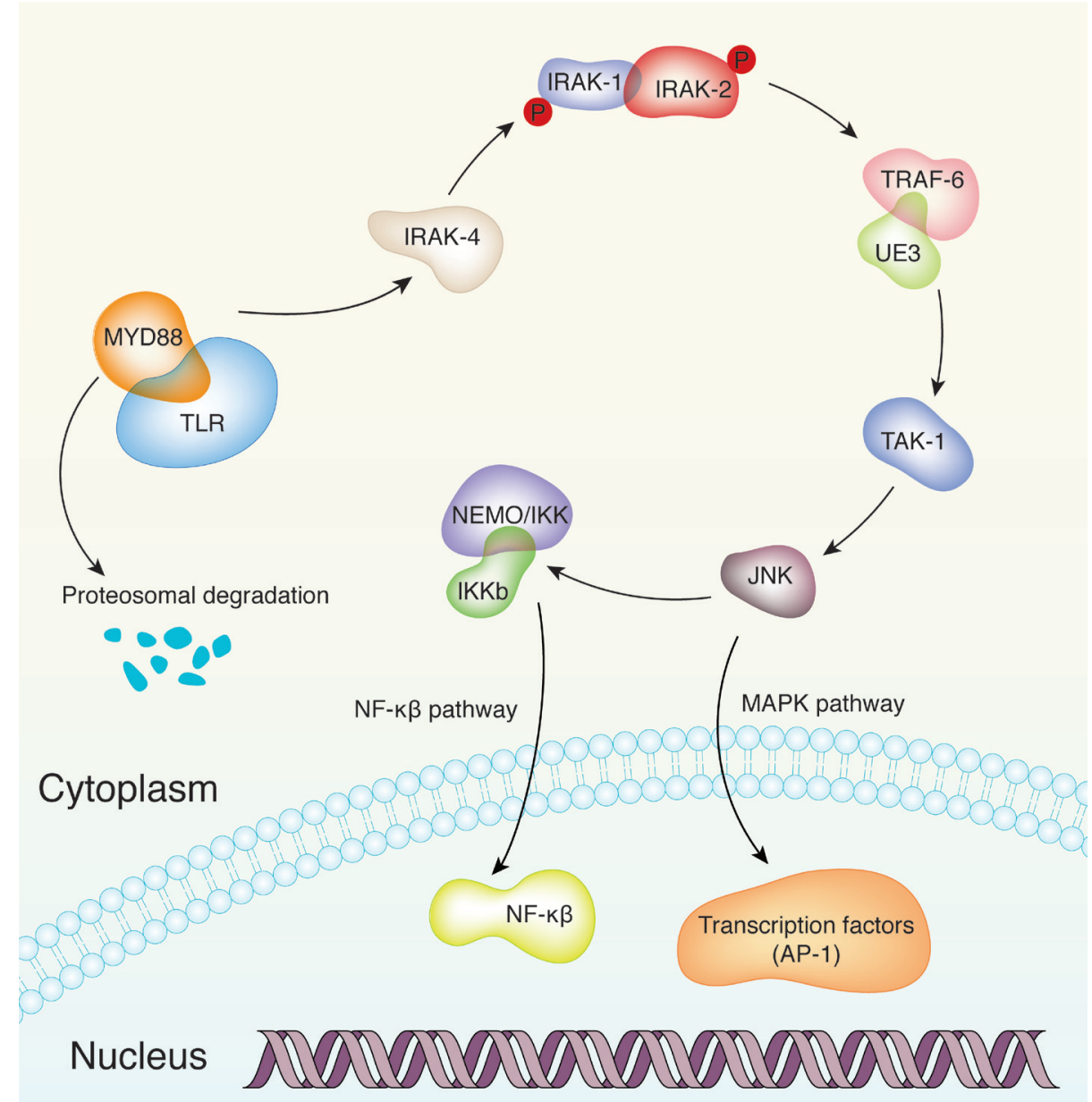

Inflammation, Immune regulation

Fig. 1. Molecules mediating the MyD88-dependent pathways. The series of events in the MyD88-dependent pathway are triggered by binding of MyD88 with TLR, which leads to activation of IRAK and TRAF proteins and ultimately stimulates the expression and activity of various transcription factors.

represents a link for the architecture of cell signaling mechanisms of the TLRs that lack catalysis. ${ }^{11}$

The mechanism of MyD88's downstream signaling is attributed to both its DD in the N-terminus and its TIR domain in the C-terminus. ${ }^{12}$ Apart from the TLRs, IL-1 has been shown to be associated with the role of MyD88 signaling as a potential inflammatory mediator. ${ }^{11}$ Considering that inflammation represents an inclination towards carcinogenesis, ${ }^{13}$ another recent study determined the pro-tumorigenic role of MyD88 in inflammation. ${ }^{11}$ TLR- and IL-1R-mediated MyD88 downstream signaling was found in another study to evoke a response in intestinal tumorigenesis. ${ }^{14}$ Moreover, MyD88 was shown to intrinsically mediate fibroblast and epithelial cell transformation ${ }^{15}$ and to be an important molecule in protection against tumor formation via the wound repairing mechanism. Finally, it has been determined that defense mechanisms involving oncogenic pathogens are promoted by the adaptive immunological reactions mediated by MyD88. ${ }^{10}$

\section{Types of MyD88 pathways}

Signaling pathways mediated by TLR exhibit two functionally distinct mechanisms: The MyD88-dependent pathway, which is common to all TLRs, and the MyD88-independent pathway, which relies on TLR3 and TLR4 signals. ${ }^{16} \mathrm{~A}$ wide array of immunological factors and receptors mediate these two pathways, as discussed below.

The MyD88-dependent pathway begins with the early stage activation of nuclear factor-kappaB (NF- $\mathrm{kB})$, which produces various proinflammatory cytokines like IL-6, IL- 8 and TNF- $\alpha$ and is involved in numerous aspects of the immunological response, cell adhesion, proliferation, angiogenesis and apoptosis (Fig. 1). ${ }^{17,18}$ Conversely, the MyD88-independent pathway replaces MyD88 with TRAM, stimulating TRIF signaling and further initiating the activation of NF- $\mathrm{KB}$ at a later stage (contrasting the early activation of the MyD88-dependent pathway), with subsequent induction of IRF3 and enhancement of IFN and IFN-inducible gene products (Fig. 2). ${ }^{19}$

\section{MyD88-dependent pathway}

The MyD88-dependent signaling pathway can be initiated by almost all of the TLRs, except for TLR3. ${ }^{20}$ The TIR domain- 


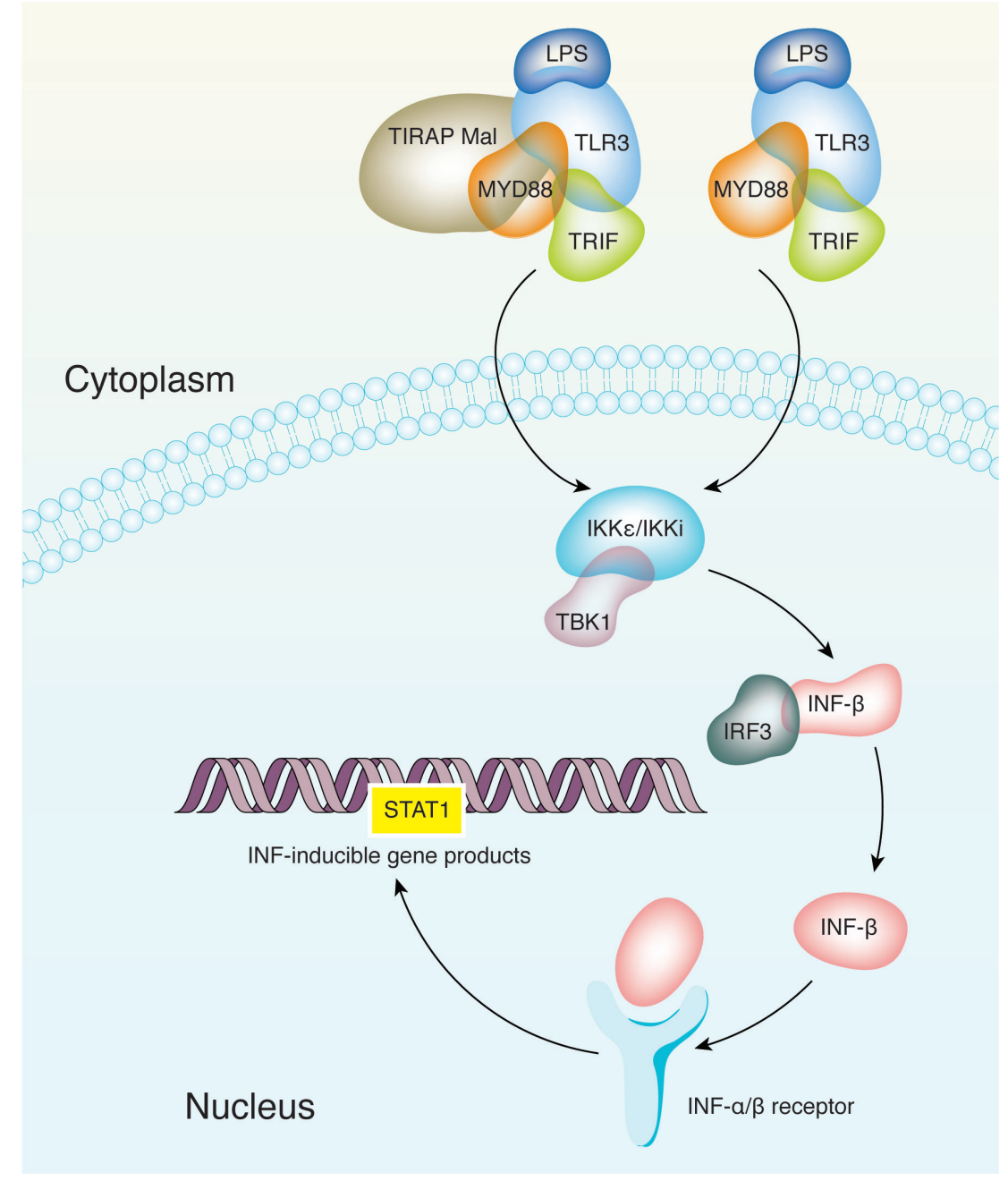

Fig. 2. Molecules mediating the MyD88-independent pathways. The cascade of events in the MyD88-indepdent pathway are initiated by interaction of the TLR and TBK proteins, with the involvement of IFN at the end stage influencing downstream gene expression.

containing adaptor protein (TIRAP)/MyD88-adaptor-like (Mal), which is the second adaptor containing a TIR domain, is known to mediate this pathway with the help of TLR2 and TLR4. A TIR domain at its $\mathrm{C}$-terminal end, together with a death domain at its $\mathrm{N}$-terminal end, is responsible for the actions of the MyD88 molecule in this pathway. It binds to TLRs via the TIR domain. Upon stimulation, MyD88 guides IRAK towards TLRs via interaction with its death domain. Next, formation of a special structure called the "Mydd-osome" occurs that is accompanied by recruitment of IRAK4 and further phosphorylation of two other kinases, IRAK1 and IRAK2. ${ }^{21}$ Once phosphorylated, IRAK becomes activated, after which it is recruited to the tumor necrosis factor receptor (TNFR)-associated factor (TRAF)6, thereby activating two distinct pathways that finally move the signal towards activation of c-Jun N-terminal kinase (JNK) and NF-кB. ${ }^{9}$

IRAK primarily functions as a serine/threonine kinase and is known to be functionally associated with the IL-1 receptor, which bears the TIR domain. ${ }^{22}$ The N-terminal DD in the IRAK protein is the primary region responsible for interaction with MyD88, but it also harbors a central kinase domain. ${ }^{9}$ Phosphorylated IRAK1 and IRAK2 are capable of interacting with E3 ubiquitin ligase and TRAF6, which occurs via the binding domain in TRAF6. ${ }^{23}$ Next,
TRAF6 ubiquitination takes place and TAK1 becomes activated, ${ }^{24}$ further initiating the dual induction of the NF- $\mathrm{KB}$ mechanism and a second pathway involving the mitogen-activated protein kinase (MAPK). ${ }^{20}$

Resting cells require NF- $\kappa \mathrm{B}$ dimers in their inactive form, held by the IкB protein in the cytoplasm. ${ }^{25}$ Proteosomal degradation follows phosphorylation of TAK1 and activation of I $\mathrm{KB}$ kinase $\mathrm{b}$ (IKKB), with phosphorylation of I $\mathrm{B}$ after NF- $\mathrm{KB}$ activation following. ${ }^{26}$

MyD88 binds to the cytoplasmic portion of TLRs, and this process is initiated by the TIR domains that interact individually. Upon stimulation, three molecules IRAK-4, IRAK-1 and TRAF6 bind with the receptor, inducing the subsequent binding of IRAK-1 to MyD88 through its DD. ${ }^{9}$ Phosphorylation of IRAK-1 is initiated by IRAK-4. The phosphorylated IRAK-1 then disassociates from TRAF6 through the receptor, after which TRAF6 interacts with a series of factors, including TAK1, TAB1 and TAB2. The assembly of TRAF6, TAK1, TAB1 and TAB2 into a larger complex leads to a further association with Ubc13 and Uev1A, thereby inducing activation of TAK $1 .{ }^{9}$

Activated TAK1 phosphorylates the IאB kinase (IKK) complex, which is a composite of IKK and NEMO/IKK, as well as mitogen- 
activated protein kinases such as $\mathrm{JNK}$; in this manner, then, the initiation of various factors involved in transcription $(\mathrm{NF}-\kappa \mathrm{B}$ and AP-1, respectively) is achieved. ${ }^{9}$ Finally, the I $\kappa \mathrm{B}$ degrades, releasing NF- $\kappa \mathrm{B}$ which then enters the nucleus and binds to $\kappa \mathrm{B}$ sites in order to activate transcription of various genes. ${ }^{27}$ Transcription factors associated with the Jun family are finally induced by the activated JNK that was initiated by the TAK1-activating MAPK pathway. ${ }^{28}$

\section{MyD88-indepdent pathway}

MyD88-independent signaling, involving TLR3, proceeds through activation of downstream signaling pathways that require TRIF as adaptor molecule and involve activation of IRF3 and production of IFN- $\beta .^{29}$ In this pathway, lipopolysaccharides act as the stimulating factor and IRF-3 is a primary transcription factor for induction of IFN.

Stat 1 activation takes place via IFN, which later stimulates the transcription of numerous IFN-mediated inducible genes. ${ }^{29-31}$ Two adaptors containing TIR domains, namely TIRAP/Mal together with the TRIF/TIR domain-containing adaptor molecule-1 (TICAM-1), have been identified by various analyses of this pathway. ${ }^{32-35}$ The signaling pathways involving TLR4, as well as TLR3, mediate this independent pathway, leading to stimulation of IRF-3 through TBK1 and IKKe/IKKi. Moreover, this independent signaling pathway is mediated by an adaptor TRIF containing a TIR domain. ${ }^{9}$

Interestingly, TRAF3 is known for exhibiting vital functions in the regulation of both the MyD88-dependent and MyD88-independent responses via the ubiquitination mechanism. The nondegradative self-ubiquitination of TRAF3 is signaled by this pathway, which promotes activation of IRF3. The MyD88-dependent signaling pathway involves TRAF3 ubiquitination-mediated breakdown, which is followed by TAK 1 stimulation. ${ }^{36}$ Henceforth, TRAF3 is the connector that links pro-inflammation with the IFNmediated response of both of these signaling pathways. ${ }^{37}$

\section{MyD88 mutations}

Mutations at the somatic cell level that confer gain-of-function to the TLR adaptor protein MYD88 have been linked to inappropriate action of TLRs, as has been demonstrated for many of the hematological malignancies. Pathogenesis associated with activated $\mathrm{B}$ cell type-diffuse large B cell lymphoma (ABC-DLBCL), an aggravated subtype of DLBCL, is solely dependent on the activation of NF- $\mathrm{kB}$ that is frequently associated with the reported mutations in MyD88. ${ }^{9}$ In a study on MyD88 mutations, around 39\% of tumor samples were found to harbor mutations in MYD88, and interestingly most of the mutations (around $29 \%$ ) alter a single nucleotide at position 265 , leading to a change from leucine to proline (L265P). ${ }^{38}$ A number of malignancies in humans have been attributed to MyD88 mutations, and cases of the L265P mutation have been recorded in almost $100 \%$ of Waldenström's macroglobulinemia (WM), in $2-10 \%$ of chronic lymphocytic leukemia (CLL), in $69 \%$ of cutaneous diffuse large B cell lymphoma (CBCL), and in $38 \%$ of primary central nervous system lymphoma (PCNSL). ${ }^{2}$ TLR activity is critical for lymphoma cells with MYD88 mutations, where its activity plays a key role and may be a target of treatment. Indeed, selective inhibition of MyD88 L265P mutation can be accomplished by endosome acidification blockade using chloroquine. ${ }^{37}$

\section{Future research prospective}

MyD88 is a comparatively new aspect of the field of cancer research, and genomic and proteomic studies may help researchers towards gaining a better understanding of the underlying biology of cancer as a whole. Proteomic study reveals the involvement of various protein molecules in signaling pathways and may better explain a disease mechanism. Such information will, in turn, assist in designing more effective, efficient and safe drugs and may open up new avenues of vaccine preparation. We propose that understanding the current pathway may reveal a lot of previously unknown information and may well be the next hot spot area of research. MyD88 signaling, in particular, may be represent an alternative cancer-associated molecule to the well-studied $\mathrm{p} 53$ pathway and may help in resolving the global cancer burden. Initial reports and findings are highly promising and one can hope that we, the worldwide scientific fraternity, can contribute meaningfully in this aspect.

\section{Conclusion}

The adaptor molecule MyD88 plays a crucial role in mediation of innate immunity signaling through the TLR and IL-1 families, which in many cancer models has provided insights into the proand anti-tumorigenic responses. ${ }^{4}$ In the scenarios of intestinal, ${ }^{14}$ liver, ${ }^{39}$ pancreatic ${ }^{40}$ and skin ${ }^{41}$ cancer progression, inflammation is regulated by signaling of MyD88. This key adaptor protein, therefore, links danger responses via TLR and various transcription factors that are known to regulate the cellular expression of a multitude of genes. ${ }^{2}$ Clearly, this adaptor protein represents a promising factor for potential therapy as it mediates a wide range of biologically vital signal transduction mechanisms associated with innate immune responses. The collective studies on this pathway are definitely a hot topic of current cancer biology research and are garnering much attention from researchers across the globe. The molecular mechanisms involving yet unrecognized genes, mutations, proteins and their interactions in cancer conditions are likely undergoing evolution, but gaining understanding of them will hopefully help the world to better understand tumorigenesis.

\section{Conflict of interest}

The authors have no conflict of interests related to this publication.

\section{Author contributions}

Making the literature survey and data collection (IB), Initiation and providing expertise in framing the manuscript (MS).

\section{References}

[1] Lord KA, Hoffman-Liebermann B, Liebermann DA. Nucleotide sequence and expression of a cDNA encoding MyD88, a novel myeloid differentiation primary response gene induced by IL6. Oncogene 1990;5:1095-1097.

[2] Wang JQ, Jeelall YS, Horikawa K. Emerging targets in human lymphoma: targeting the MYD88 mutation. Blood and Lymphatic Cancer: Targets and Therapy 2013;3:53-61. doi:10.2147/BLCTT.S35292.

[3] Hultmark D. Macrophage differentiation marker MyD88 is a member of the Toll/IL-1 receptor family. Biochem Biophys Res Commun 1994;199:144-146. doi:10.1006/bbrc.1994.1206 
[4] Kennedy CL, Najdovska M, Tye H, McLeod L, Yu L, Jarnicki A, et al. Differential role of MyD88 and Mal/TIRAP in TLR2-mediated gastric tumourigenesis. Oncogene 2014;33:2540-2546. doi:10.1038/onc.2013.205.

[5] O'Neill LA. Therapeutic targeting of Toll-like receptors for inflammatory and infectious diseases. Curr Opin Pharmacol 2003;3:396-403. doi:10.1016/S14714892(03)00080-8.

[6] Warner N, Núñez G. MyD88. A Critical Adaptor Protein in Innate Immunity Signal Transduction. J Immunol 2013;190:3-4. doi:10.4049/jimmunol.1203103.7.

[7] Chuffa LG, Fioruci-Fontanelli BA, Mendes LO, Ferreira Seiva FR, Martinez M, Fávaro WJ, et al. Melatonin attenuates the TLR4-mediated inflammatory response through MyD88- and TRIF-dependent signaling pathways in an in vivo model of ovarian cancer. BMC Cancer 2015;15:34. doi:10.1186/s12885015-1032-4.

[8] Piras V, Selvarajoo K. Beyond MyD88 and TRIF pathways in Toll-like receptor signaling. Front Immunol 2014;5:70. doi:10.3389/fimmu.2014.00070.

[9] Takeda K, Akira S. TLR signaling pathways. Seminars in Immunology 2004;16:3-9. doi:10.1016/j.smim.2003.10.003.

[10] Salcedo R, Cataisson C, Hasan U, Yuspa SH, Trinchieri G. MyD88 and its divergent toll in carcinogenesis. Trends Immunol 2013;34:379-89. doi:10.1016/j. it.2013.03.008.

[11] Coste I, Le Corf K, Kfoury A, Hmitou I, Druillennec S, Hainaut P, et al. Dual function of MyD88 in RAS signaling and inflammation, leading to mouse and human cell transformation. J Clin Invest 2010;120:3663-3667. doi:10.1172/ JCI42771.

[12] Lee MS, Kim YJ. Signaling pathways downstream of pattern-recognition receptors and their cross talk. Annu Rev Biochem 2007;76:447-480. doi:10.1146/ annurev.biochem.76.060605.122847.

[13] Clevers H. At the crossroads of inflammation and cancer. Cell 2004;118:671674. doi:10.1016/j.cell.2004.09.005.

[14] Rakoff-Nahoum S, Medzhitov R. Regulation of spontaneous intestinal tumorigenesis through the adaptor protein MyD88. Science. 2007;317:124-127. doi: $10.1126 /$ science. 1140488 .

[15] Swann JB Vesely MD, Silva A, Sharkey J, Akira S, Schreiber RD, Smyth MJ, et al. Demonstration of inflammation induced cancer and cancer immunoediting during primary tumorigenesis. Proc Natl Acad Sci U S A 2008;105:652-656. doi:10.1073/pnas.070859410516.

[16] Akira S, Takeda K, Kaisho T. Toll-like receptors: critical proteins linking innate and acquired immunity. Nat Immunol 2001;2:675-680. doi:10.1038/9060917.

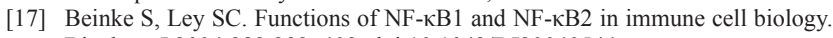
Biochem J 2004;382:393-409. doi:10.1042/BJ20040544.

[18] Akira S. Toll-like receptor signaling. J Biol Chem 2003;278:38105-38108. doi:10.1074/jbc.R300028200.

[19] Akira S, Takeda K. Toll-like receptor signalling. Nat Rev Immunol 2004;4:499 511. doi:10.1038/nri1391.

[20] Compagno M, Lim WK, Grunn A, Nandula SV, Brahmachary M, Shen Q, et al. Mutations of multiple genes cause deregulation of NF-kappaB in diffuse large B-cell lymphoma. Nature 2009;459:717-721. doi:10.1038/nature07968.

[21] Lin SC, Lo YC, Wu H. Helical assembly in the MyD88-IRAK4-IRAK2 complex in TLR/IL-1R signaling. Nature 2010;465:885-890. doi:10.1038/nature09121.

[22] Häcker H, Vabulas RM, Takeuchi O, Hoshino K, Akira S, Wagner H. Immune cell activation by bacterial CpG-DNA through myeloid differentiation marker 88 and tumor necrosis factor receptor-associated factor (TRAF)6. J Exp Med 2001;192:595-600. doi:10.1084/jem.192.4.595.

[23] Ye H, Arron JR, Lamothe B, Cirilli M, Kobayashi T, Shevde NK, et al. Distinct molecular mechanism for initiating TRAF6 signaling. Nature 2002;418:443447. doi:10.1038/nature00888.
[24] Xia ZP, Sun L, Chen X, Pineda G, Jiang X, Adhikari A, et al. Direct activation of protein kinases by unanchored polyubiquitin chains. Nature 2009;461:114 119. doi: 10.1038 /nature 08247

[25] Jacobs MD, Harrison SC. Structure of an $\mathrm{I} \kappa \mathrm{B} \alpha / \mathrm{NF}-\kappa \mathrm{B}$ Complex. Cell 1998;95:749-758. doi:10.1016/S0092-8674(00)81698-0.

[26] Li Q, Lu Q, Bottero V, Estepa G, Morrison L, Mercurio F, et al. Enhanced NFkappaB activation and cellular function in macrophages lacking IkappaB kinase 1 (IKK1). Proc Natl Acad Sci U S A 2005;102:12425-12430. doi:10.1073/ pnas.0505997102.

[27] Solt LA, May MJ. The IkappaB kinase complex: master regulator of NF-kappaB signaling. Immunol Res 2008:42:3-18. doi:10.1007/s12026-008-8025-1.

[28] Liu W, Ouyang X, Yang J, Liu J, Li Q, Gu Y, et al. AP-1 activated by toll-like receptors regulates expression of IL-23 p19. J Biol Chem 2009;284:2400624016. doi:10.1074/jbc.M109.025528.

[29] Doyle SE, Vaidya SA, O'Connell R, Dadgostar H, Dempsey PW, Wu TT, et al. IRF3 mediates a TLR3/TLR4-specific antiviral gene program. Immunity 2002;17:251-263. doi:10.1016/S1074-7613(02)00390-4.

[30] Toshchakov V, Jones BW, Perera PY, Thomas K, Cody MJ, Zhang S, et al. TLR4, but not TLR2, mediates IFN-beta-induced STAT1alpha/beta-dependent gene expression in macrophages. Nat Immunol 2002;3:392-398. doi:10.1038/ ni774.

[31] Hoshino K, Kaisho T, Iwabe T, Takeuchi O, Akira S. Differential involvement of IFN-in Toll-like receptor-stimulated dendritic cell activation. Int Immunol 2002;14:1225-1231. doi:10.1093/intimm/dxf089.

[32] Horng T, Barton GM, Medzhitov R. TIRAP: an adapter molecule in the Toll signaling pathway. Nat Immunol 2001;2:835-841. doi:10.1038/ni0901-835.

[33] Fitzgerald KA, Palsson-McDermott EM, Bowie AG, Jefferies CA, Mansell AS, Brady G, et al. Mal (MyD88-adapter-like) is required for Toll-like receptor-4 signal transduction. Nature 2001;413:78-83. doi:10.1038/35092578.

[34] Yamamoto M, Sato S, Mori K, Hoshino K, Takeuchi O, Takeda K, et al. Cutting edge: a novel Toll/IL-1 receptor domain-containing adapter that preferentially activates the IFN-betapromoter in the Toll like receptor signaling. J Immunol 2002;169:6668-6672. doi:10.4049/jimmunol.169.12.6668.

[35] Oshiumi H, Matsumoto M, Funami K, Akazawa T, Seya T. TICAM-1, an adaptor molecule that participates in Toll-like receptor 3-mediated interferon-beta induction. Nat Immunol 2003;4:161-167. doi:10.1038/ni886.

[36] Tseng PH, Matsuzawa A, Zhang W, Mino T, Vignali DA, Karin M. Differen modes of ubiquitination of the adaptor TRAF3 selectively activate the expression of type I interferons and proinflammatory cytokines. Nat Immunol 2010;11:70-75. doi:10.1038/ni.1819.

[37] Wang JQ, Jeelall YS, Beutler B, Horikawa K, Goodnow CC. Consequences of the recurrent MYD88(L265P) somatic mutation for B cell tolerance. J Exp Med 2014;211:413-426. doi:10.1084/jem.20131424.

[38] Ngo VN, Young RM, Schmitz R, Jhavar S, Xiao W, Lim KH, et al. Oncogenically active MYD88 mutations in human lymphoma. Nature 2011;470:115119. doi:10.1038/nature09671.

[39] Naugler WE, Sakurai T, Kim S, Maeda S, Kim K, Elsharkawy AM, et al. Gender disparity in liver cancer due to sex differences in MyD88-dependent IL-6 production. Science 2007;317:121-124. doi:10.1126/science.1140485.

[40] Ochi A, Nguyen AH, Bedrosian AS, Mushlin HM, Zarbakhsh S, Barilla R, et al. MyD88 inhibition amplifies dendritic cell capacity to promote pancreatic carcinogenesis via Th2 cells. J Exp Med 2012;209:1671-1687. doi:10.1084/ jem.20111706.

[41] Cataisson C, Salcedo R, Hakim S, Moffitt BA, Wright L, Yi M, et al. IL-1RMyD88 signaling in keratinocyte transformation and carcinogenesis. J Exp Med 2012;209:1689-1702. doi:10.1084/jem.20101355. 